\title{
Tubeless Percutaneous Nephrolithotomy: Can be a Choice, Why Not?
}

\author{
Mert Ali Karadag ${ }^{*}, 1$, Kursat Cecen ${ }^{1}$, Aslan Demir ${ }^{1}$, Ramazan Kocaaslan ${ }^{1}$, Kerem Taken ${ }^{2}$ and \\ Fatih Altunrende ${ }^{3}$
}

\author{
${ }^{1}$ Kafkas University Faculty of Medicine, Department of Urology, Kars, Turkey \\ ${ }^{2}$ Yuzuncuyil University Faculty of Medicine, Department of Urology, Van, Turkey \\ ${ }^{3}$ Bilim University Faculty of Medicine, Department of Urology, Istanbul, Turkey
}

\begin{abstract}
Percutaneous nephrolithotomy (PCNL) has been widely accepted and is commonly used to treat renal calculi. The optimal drainage of kidney after PCNL has not been clearly determined yet. Placement of an $18 \mathrm{~F}$ to $24 \mathrm{~F}$ nephrostomy tube at the end of the procedure is accepted as standard of care to date. The main advantages are adequate renal drainage, hemostatic tamponade and providing renal access for second look PCNL. However, based on the concept that the purpose of the tube is only to maintain adequate drainage of the kidney, a "tubeless" approach has been developed by placing a ureteral stent or catheter to provide drainage after PCNL instead of a nephrostomy tube. Tubeless PCNL is an effective and safe procedure for treatment of renal stones in selected cases. This procedure can even be chosen for patients with previous renal surgery, and hemorrhagic tendency. By using this method, less postoperative pain and a shorter hospital stay can be achieved, when compared with conventional PCNL. There is a controversy over ideal drainage system after PCNL in recent years. Herein, we made a systematic review for efficacy and safety of tubeless PCNL, totally tubeless PCNL, discussed different variations and compared the outcomes of this technique with standart PCNL.
\end{abstract}

Keywords: Complications, percutaneous renal surgery, renal stones, totally tubeless, tubeless percutaneous nephrolithotomy, urolithiasis.

\section{BACKGROUND}

Percutaneous nephrolithotomy (PCNL) has been widely accepted and commonly used to treat renal calculi [1]. The optimal drainage of kidney after PCNL has not been clearly determined yet. Placement of an $18 \mathrm{~F}$ to $24 \mathrm{~F}$ nephrostomy tube at the end of the procedure is accepted as standart of care to date. The main advantages are adequate renal drainage, hemostatic tamponade and providing renal access for second look PCNL.

However, based on the concept that the purpose of the tube is only to maintain adequate drainage of the kidney, a "tubeless" approach has been developed by placing a ureteral stent or catheter to provide drainage after PCNL instead of a nephrostomy tube.

Today, several studies are stating the superiority of tubeless PCNL over conventional PCNL in terms of less morbidity, lower postoperative pain and a shorter hospital stay [2-5].

\section{AIM}

As a result, there is a controversy over ideal drainage system after PCNL in recent years. Here, we made a systematic review for efficacy and safety of tubeless PCNL, totally tubeless PCNL, discussed different variations and compared the outcomes of this technique with standart PCNL.

*Address correspondence to this author at the Kafkas University Faculty of Medicine, Department of Urology, Kars, Turkey; Tel: +90 53255843 24;

E-mail: karadagmert@yahoo.com

\section{MATERIALS AND METHODS}

Randomized or quasi-randomized controlled trials and retrospective studies having high number of patients were selected from the following sources: Cochrane Central Register of Controlled Trials (CENTRAL), medline, EMBASE, pubmed et al. These heading terms were used for searching the studies: percutaneous nephrolithotomy, tubeless percuta-neous nephrolithotomy, tubeless, nephrostomy tube or nephrostomy drainage. The retrieval time ended in April 2013.

\section{RESULTS}

\section{History of Tubeless PCNL}

In 1984, Wickham et al. first published the results of 100 patients who underwent PCNL in whom no ureteral catheter, no stent or no nephrostomy tubes were used. They concluded that, this approach was safe and efficient with shorter hospital stay $(<24$ hours) [6]. In 1997, Bellman et al. reported the results of 20 patients with small stone burdens who underwent tubeless PCNL [7]. The authors stated that this technique was uncomplicated and had the advantages of less hospitalization time and decreased analgesic requirements.

\section{Totally Tubeless PCNL}

As firstly described by Wickham et al., another technical variation of tubeless PCNL is totally tubeless approach [6]. They concluded that if the operated kidney was stone free, collecting system was intact and there wasn't any excessive 
bleeding, there was no need for nephrostomy drainage [6]. However, in 1986, Winfield et al. published the complications of 2 patients who had undergone a PCNL operation for simple upper tract calculi and early nephrostomy tube removal [8]. They experienced serious hemorrhage and urinary extravasation, urinoma requiring internal stenting, transfusion and prolonged hospitalization. This study was a cornerstone for the consideration of nephrostomy tube drainage should be provided during the first 24 to 48 hours after PCNL.

Today, there are few successful reports of totally tubeless PCNL. They mentioned that the hospitalization time, return to normal activities and analgesia requirements were significantly less in totally tubeless group, when compared with conventional PCNLs [9-11].

This approach was applied by Aghamir et al. for patients having renal anomalies like horseshoe kidneys, rotational anomalies and ectopic kidneys [12]. The differences between tubeless and standart PCNL groups in terms of operation time, transfusion rates, complications, retreatment and overall stone free rate were not statistically significant. The hospitalization time, return to nomal activities and analgesia requirements were statistically lower in totally tubeless group.

In a recent study, same group assessed the outcome and safety of the totally tubeless PCNL in patients with renal stones in the upper pole of the kidney and subcostal access [13]. Seventy patients with upper pole renal stones were enrolled in this study. Stone sizes were over $1.5 \mathrm{~cm}$. All the stones were extracted through successful subcostal accesses. They stated that totally tubeless PCNL for the upper pole renal stone via subcostal access was accompanied by decreased hospital stay and analgesics use and a rapid return to normal activity.

These studies suggested that the best drainage of the kidney was the normal peristalting ureter. However, this approach has not been accepted worldwide, due to obstruction chance of the ureter with stone fragments or blood clots after stone extraction. Most centers prefer some kind of internal drainage after tubeless procedures.

\section{Tubeless PCNL vs Conventional PCNL}

To the best of our knowledge, there are only 3 studies in the literature comparing tubeless PCNL with standart nephrostomy drainage in a randomized fashion $[2,3,14]$. Desai et al. reported the results of a study that compared large and small nephrostomy drainage tubes with tubeless PCNL [2]. The main inclusion criteria was single subcostal access and absence of previous surgery on the ipsilateral renal unit. There were 10 cases in each study group. The stone burden ranged between $243 \mathrm{~mm}^{2}$ and $264 \mathrm{~mm}^{2}$. Marcovich et al. likewise compared large and small nephrostomy tubes and tubeless PCNL in a randomized study [3]. There were 20 patients in each study group, and the main exclusion criteria were previous surgery on the ipsilateral renal unit and the need for supracostal puncture. The stone sizes were between 3 and $3.6 \mathrm{~cm}$. Feng and colleagues compared the results of standart PCNL, mini-PCNL, and tubeless PCNL. The number of patients in each study group ranged from 8 to 10 [14]. Similar to previously reported studies, their exclusion criteria was patients having more than 2 access tracts. The stone burden ranged between 4.38 and $8.36 \mathrm{~cm}^{2}$. Marcovich et al. could not find any overwhelming advantage of any drainage system over the others [3]. The other 2 studies demonstrated that tubeless PCNL was associated with the less postoperative pain and urinary leakage, shorter hospital stay, and lower morbidity $[2,14]$.

\section{Tubeless PCNL in Obese Patients}

Yang et al. reported safe and effective tubeless percutaneous renal surgery in obese and morbidly obese patients [15]. They analysed the clinical data of a subset of patients who were considered normal weight (Body Mass Index (BMI): 18.5-25), overweight (BMI: 25-30), obese (BMI: 30-40) and morbidly obese (BMI $>40)$. Of these patients, $5(3.8 \%)$ were morbidly obese, $28(21.2 \%)$ were obese, $55(41.4 \%)$ were considered overweight. The influence of BMI on the transfusion rates, days of hospitalization, and stone free outcome was compared. The stone group did not demonstrate statistically significant relationships between $\mathrm{BMI}$ and transfusion rate, length of hospitalization and stone free rate.

\section{Tubeless PCNL Choice}

Most studies focused on choosing tubeless PCNL only in selected patients with uncomplicated stones $[6,7,16]$. The inclusion criteria for tubeless PCNL was a single renal access (not supracostal), stone burden $<3 \mathrm{~cm}$, operation time less than 2 hours, no significant perforations and bleeding, no requirement for second look procedure and complete clearance of stones.

\section{Tubeless PCNL After Supracostal Access?}

In 2007, Sofikerim et al. published the results of 48 patients who underwent PCNL via supracostal accesses [17]. The patients were randomized to either have an $18 \mathrm{~F}$ re-entry nephrostomy tube (Group 1) or a $6 \mathrm{~F}$ double J (D-J) stent (Group $2)$. The two groups were well matched for age, sex, stone size, stone laterality, and number of previous renal procedures. Postoperative visual analog pain scale (VAS) scores at 8, 24 hours and 14 days after surgery, analgesic use in hospital, length of hospital stay, success rate, blood transfusion rate and postoperative complications were compared for 2 groups. The group 2 had statistically significant decreased hospital stay, lower analgesic requirement and VAS scores at 8 and 24 hours after surgery. The rate of blood transfusion in the 2 groups was similar. There was no difference between the groups in VAS scores on postoperative day 14. Interestingly, the number of supracostal accesses was significantly higher in group 2 than group 1. There was no urine leakage or formation of urinoma in patients with D-J stents. The authors concluded that tubeless PCNL was safe and effective even after supracostal access and was associated with less postoperative pain and a shorter hospital stay.

\section{Tubeless PCNL in Children}

In a recent study, Bilen et al. analysed the outcomes of tubeless mini percutaneous nephrolithotomy in infants and preschool children, and compared them with age matched controls, who underwent nephrostomy drainage [18]. A total of 28 renal units in 26 children were operated for stone disease using the mini percutaneous nephrolithotomy. Children who 
were stone free and had a clear nephrostomy tract were inserted ureteral catheters. Those with residual stones or bleeding from the nephrostomy tract underwent nephrostomy drainage. Both groups were compared in terms of patient and stone characteristics, post operative findings. They found that surgery and fluoroscopy times were shorter in the tubeless group. Complication rates were higher and hospital stay was longer in the nephrostomy group. Tubeless group had a $91.6 \%$ stone free rate, whereas nephrostomy group had a rate of $78.5 \%$. According to the results, they concluded that tubeless percutaneous nephrolithotomy could be a safe option for selected children with renal stone disease. Patient selection like low stone volume and infection free stones that were removed completely without bleeding was the most important factor for safety and success of the procedure.

Salem and colleagues assessed the effectiveness of tubeless PCNL in 20 children with an average age of 7.5 years [19]. There was no significant bleeding intra or postoperatively. They concluded that tubeless PCNL had the advantages of lower hospital stay and pain, when compared with a group of 10 children having similar characteristics with nephrostomy drainage.

\section{Tubeless PCNL in Obese Patients}

Yang et al. reported safe and effective tubeless percutaneous renal surgery in obese and morbidly obese patients [15]. They analysed the clinical data of a subset of patients who were considered normal weight (Body Mass Index (BMI): 18.5-25), overweight (BMI: 25-30), obese (BMI: 30-40) and morbidly obese (BMI $>40)$. Of these patients, $5(3.8 \%)$ were morbidly obese, $28(21.2 \%)$ were obese, $55(41.4 \%)$ were considered overweight. The influence of BMI on the transfusion rates, days of hospitalization, and stone free outcome was compared. The stone group did not demonstrate statistically significant relationships between BMI and transfusion rate, length of hospitalization and stone free rate.

\section{Tubeless PCNL with Previous Open Surgery}

Shah et al. published the results of 25 patiens with a history of ipsilateral open surgery who underwent tubeless PCNL [20]. Their exclusion criteria was cases requiring more than 2 accesses, significant bleeding, and a significant residual stone burden that required a second look PCNL. The perioperative outcome of these patients was retrospectively compared with same number of patients having conventional PCNL with previous open renal surgery. They found that tubeless group required less analgesics and had a 10 hour earlier discharge. They concluded that this technique was safe and had the advantages even in cases with a history of open renal surgery.

\section{Safety of Tubeless PCNL in Patients with Hemorrhagic Diatesis}

Jou and colleagues reported their experience of performing tubeless PCNL in patients with cirrhosis and on antiplatelet therapy [21]. At the end of the procedure, all the bleeding points were cauterized through renal access for hemostasis. There were 16 patients having antiplatelet therapy, and 6 were cirrhotic. The average hospital stay was 3.8 days and the stone free rate was $87.5 \%$. There was no uncontrolled hemorrhage during and after the operation, only one patient required blood transfusion postoperatively. No patient experienced any thromboembolic complications. Finally, they suggested that with careful hemostasis, tubeless PCNL procedure can be applied to patients having bleeding tendency.

\section{Tubeless PCNL in Patients with Staghorn Calculi}

The feasibility of tubeless PCNL was investigated in a very recent study from Korea [22]. The aim of the study was to investigate and compare the outcomes of conventional PCNL and tubeless PCNL in patients with staghorn calculi. The study had a retrospective nature and included a period of 9 years. A total of 165 patients were enrolled in the study. Conventional approach was preferred for 106 patients and tubeless for 59 . There were not any significant differences between two groups in terms of sex, age, BMI, stone laterality. The mean stone burden of conventional and tubeless approach was $633.6 \pm 667.4$ $\mathrm{mm}^{2}$ and $529.9 \pm 362.8 \mathrm{~mm}^{2}$, respectively. They revealed a stone free rate of $78 \%$ in patients who underwent tubeless PCNL; but this value was $69.8 \%$ in patients who underwent conventional approach. This difference was not statistically significant. The interesting point was that when the complications like fever, bleeding, infection between 2 groups were compared, no significant differences were encountered. They concluded that tubeless PCNL in patients with staghorn calculi had the same outcomes of conventional approach and could be preferred in this patient population. For prevention of hemorrhage, the investigators placed 2 pieces of Cutanplast (Mascia Brunelli, Italy) via the nephrostomy tract at the end of tubeless procedures.

\section{The Usage of Hemostatic Agents in Tubeless PCNL}

The outcomes and efficacy of autologous fibrin sealant usage in tubeless PCNL was investigated in a study [23]. The investigation was a prospective study and included 43 patients who underwent tubeless PCNL. The autologous fibrin sealant was used in 15 patients and the other 28 was not. The peroperative parameters like mean stone burden, sex, age, laterality were statistically insignificant. The mean postoperative hemoglobin value of patients with autologous fibrin sealant was $12.93 \pm 1.43 \mathrm{gr} / \mathrm{dl}$ and same value was $12.54 \pm 1.55 \mathrm{gr} / \mathrm{dl}$ for patients without the sealant. The only statistically significant difference between two groups was mean catheter duration. The mean catheter duration of patients with fibrin sealant was statistically lower than the others. They concluded that autologous fibrin sealant did not affect the outcomes of tubeless PCNL; but its use in PCNL was safe.

The usage of a new antihemostatic agent called as Ankaferd Blood StopperTM (ABS) was investigated in a recent study [24]. The study was prospective and included 90 patients. There were 2 groups, group 1 involved the patients in whom ABS was used and the other not. Mean age, stone size, access number, serum creatinine change, operation time, renal thickness, VAS score and hospitalization time were not statistically different between 2 groups. Albeit the nephroscope time was longer in ABS group, hemoglobin decrease and urine clarity time were statistically lower when compared with the other group. Hemoglobin decrease was $1.40 \pm 1.04 \mathrm{gr} / \mathrm{dl}$ in ABS group; but this value was $1.84 \pm 1.15 \mathrm{gr} / \mathrm{dl}$ in control group $(\mathrm{p}=0.034)$. The urine clarity of ABS and control group was $9.60 \pm 5.50$ hours and 
$11.95 \pm 4.71$ hours, respectively $(p=0.012)$. They concluded that ABS was an efficient and reliable hemostatic agent in tubeless PCNL.

Tachosil $^{\circledR}$ was the another antihemostatic agent that was used in tubeless PCNL tract similarly with local anesthetic molecules that were used for relief of pain [25]. Its efficacy in reducing urine leakage and bleeding was investigated in a prospective, randomized trial [26]. A total of 100 patients who underwent PCNL were randomized to either receive a $16 \mathrm{Fr}$ nephrostomy tube (Group 1) or Tachosil in the tract (Group 2). All of the patients were inserted $d-j$ stents. They revealed that tract related complications were significantly more common in group $1(25.5 \%$ vs $2 \%, \mathrm{p}<0.001)$. Albeit the urinary leakage in group 1 was statistically higher than group $2(19.1 \%$ vs $2 \%$, $\mathrm{p}=0.007)$, the perirenal hematoma formation was not $(6.4 \% \mathrm{vs}$ $0 \%, p=0.113)$. There was not a statistically significant difference among the groups in terms of analgesic doses and VAS scores. The authors concluded that even failed in reducing pain and analgesic requirement, Tachosil ${ }^{\circledR}$ provided better tract control and shorter hospital stay than the group with nephrostomy tube.

\section{CONCLUSION}

Tubeless PCNL is an effective and safe procedure for treatment of renal stones in selected cases. This procedure can even be chosen for patients with previous renal surgery, hemorrhagic tendency, high BMI, staghorn calculi and upper pole stones. By using this method, less postoperative pain and a shorter hospital stay can be achieved, when compared with conventional PCNL. We think that these results should encourage urologists for choice of tubeless PCNL in selected cases. In the future, tubeless approach may be more palatable to patients than standart PCNL in terms of less hospital stay and lower analgesics requirement.

\section{CONFLICT OF INTEREST}

The authors confirm that this article content has no conflict of interest.

\section{ACKNOWLEDGEMENT}

Declared none.

\section{REFERENCES}

[1] Matlaga BR, Kim SC, Lingeman JE. Improving outcomes of percutaneous nephrolithotomy: access. EAU Update Series 2005; 3: 37-43.

[2] Desai MR, Kukreja RA, Desai MM, et al. A prospective randomized comparison of type of nephrostomy drainage following percutaneous nephrostolithotomy: large bore versus small bore versus tubeless. J Urol 2004; 172: 565-7.

[3] Marcovich R, Jacobson AI, Singh J, et al. No panecea for drainage after percutaneous nephrolithotomy. J Endourol 2004; 18: 743-7.
[4] Tefekli A, Altunrende F, Tepeler K, Tas A, Aydin S, Muslumanoglu AY. Tubeless percutaneous nephrolithotomy in selected patients: a prospective randomized comparison. Int Urol Nephrol 2007; 39: 57-61.

[5] Akman T, Binbay M, Yuruk E, et al. Tubeless procedure is most important factor in reducing length of hospitalization after percutaneous nephrolithotomy: results of unvariable and multivariable models. Urology 2011; 77: 299-304.

[6] Wickham JE, Miller RA, Kellett MJ, Payne SR. Percutaneous nephrolithotomy: one stage or two? Br J Urol 1984; 56: 582-5.

[7] Bellman GC, Davidoff R, Candela J, Gerspach J, Kurtz S, Stout L. Tubeless percutaneous renal surgery. J Urol 1997; 157: 1578-82.

[8] Winfield HN, Weyman P, Clayman RV. Percutaneous nephrostolithotomy: complications of premature nephrostomy removal J Urol 1986; 136: 77-9.

[9] Bdesha AS, Jones CR, North EA, Pinfield J, Boyd PJ. Routine placement of a nephrostomy tube is not necessary after percutaneous nephrostolithotomy. Br J Urol 1997; 79: 1-4.

[10] Karami H, Gholamrezaie HR. Totally tubeless nephrolithotomy in selected patients. J Endourol 2004; 18: 475-6.

[11] Aghamir SM, Hosseini SR, Gooran S. Totally tubeless percutaneous nephrolithotomy. J Endourol 2004; 18: 647-8.

[12] Aghamir SM, Mohammadi A, Mosavibahar SH, Meysamie AP. Totally tubeless percutaneous nephrolithotomy in renal anomalies. J Endourol 2008; 22: 2131-34.

[13] Aghamir SM, Modaresi SS, Aloosh M, Tajik A. Totally tubeless percutaneous nephrolithotomy for upper pole renal stone using subcostal access. J Endourol 2011; 25: 583-6.

[14] Feng MI, Tamaddon K, Mikhail A, Kaptein JS, Bellman GC. Prospective randomized study of various techniques of percutaneous nephrolithotomy. Urology 2001; 58: 345-50.

[15] Yang RM, Bellman GC. Tubeless percutaneous renal surgery in obese patients. Urology 2004; 63: 1036-40.

[16] Limb J, Bellman GC. Tubeless percutaneous renal surgery: review of first 112 patients. Urology 2002; 59: 527-31.

[17] Sofikerim M, Demirci D, Huri E, Ersekerci E, Karacagil M. Tubeless percutaneous nephrolithotomy: safe even in supracostal access. J Endourol 2007; 21: 967-71.

[18] Bilen CY, Gunay M, Ozden E, Inci K, Sarikaya S, Tekgul S. Tubeless mini percutaneous nephrolithotomy in infants and preschool children: a preliminary report. J Urol 2010; 184: 2498-502.

[19] Salem HK, Morsi HA, Omran A, Daw MA. Tubeless percutaneous nephrolithotomy in children. J Pediatr Urol 2007; 3: 235-8.

[20] Shah HN, Mahajan AP, Hegde SS, Bansal M. Tubeless percutaneous nephrolithotomy in patients with previous ipsilateral open surgery: a feasibility study with review of the literature. J Endourol 2008; 22: 1924.

[21] Jou YC, Shen CH, Lin CT, Cheng MC, Chen PC, Tsai YS. Safety and efficacy of tubeless percutaneous nephrolithotomy in patients on antiplatelet therapy and cirrhotic patients. Urol Res 2011; 39: 393-6.

[22] Lee SC, Kim CH, Kim KT, et al. Is tubeless percutaneous nephrolithotomy a feasible technique for the treatment of staghorn calculi? Korean J Urol 2013; 54: 693-6.

[23] Ziaee SAM, Sarhangnejad R, Abolghasemi H, et al. Autologous fibrin sealant in tubeless percutaneous nephrolithotomy; a prospective study. Urol J 2013; 10(3): 999-1003.

[24] Istanbulluoglu MO, Kaynar M, Cicek T, Kosan M, Ozturk B, Ozkardes H. A new hemostatic agent (Ankaferd Blood Stopper $\left({ }^{\circledR}\right)$ ) in tubeless percutaneous nephrolithotomy: a prospective randomized study. J Endourol 2013; 27: 1126-30.

[25] Kirac M, Tepeler A, Bozkurt OF, et al. The efficacy of bupivacaine infiltration on the nephrostomy tract in tubeless and standard percutaneous nephrolithotomy: a prospective, randomized, multicenter study. Urology 2013; 82: 526-31.

[26] Cormio L, Perrone A, Di Fino G, et al. Tachosil $\left({ }^{\circledR}\right)$ sealed tubeless percutaneous nephrolithotomy to reduce urine leakage and bleeding: outcome of a randomized controlled study. J Urol 2012; 188: 145-50. 